\title{
Improved Approximate Gradient Method in MPPT Control of Photovoltaic Generation System
}

\author{
Chen Zhou, Xi Ziqiang, Lu bin \\ School of Electronic \& Electronic Engineering, Hubei University of Technology; Hubei Collaborative \\ Innovation Center for High-efficient Utilization of Solar Energy, Hubei University of Technology, \\ Wuhan, China \\ 1186912704@qq.com, Xizq60@sina.com,@xyz.com,1817605122@qq.com
}

Keywords: approximate gradient; MPPT; photovoltaic system

\begin{abstract}
Approximate gradient method is improved for the maximum power point tracking (MPPT) of photovoltaic generation system. The improvements are implemented by: (1) applying constant voltage tracking method to narrow the search range when external conditions change drastically; (2) adding constraint conditions to optimize the power curve. Based on the simulation of the original and improved approximate gradient methods, it is shown that the improved method ameliorates the tracking performance by avoiding the defeats as "divide by zero error", tracking failure and oscillation.
\end{abstract}

\section{Introduction}

Since the output characteristics of photovoltaic (PV) array is nonlinear and influenced largely by environmental factors, the maximum power point tracking (MPPT) [1] of the PV array is essential in the PV generation system [2]. As a result, many MPP tracking (MPPT) methods have been developed and implemented. The constant voltage tracking (CVT), which keeps the output voltage as 75\% of open-circuit current $\left(V_{o c}\right)$, is one of the simplest methods for MPPT. However, CVT has some disadvantages, such as low control precision and large power loss, which is because of the empirical MPP data of $75 \% V_{O C}$, and the ignorance of the impact of temperature, thus making the working point tracked by CVT is not the true MPP. The most widely used MPPT method is perturbation and observation (P\&O) method, which adjusts the output voltage with small voltages called perturbation steps to find MPP. In traditional P\&O method, the step size is a fixed value that there are oscillations near the MPP. Although a smaller step size can reduce the oscillation, it needs heavy computational time and be easy to fail when atmospheric conditions rapidly changes [3-5].

To optimize the perturbation step by $\mathrm{P} \& \mathrm{O}$ method, the optimal gradient method, using the negative gradient direction of the objective function as the search direction to approach the minimum function, analyzes the power-voltage (P-V) curves to find the optimal gradient at the working point and then changes the size or direction of perturbation step to make $d P$ / $d V$ close to 0 . The optimal gradient method is a high precision optimization algorithm completely based on mathematical derivation. However, it has two drawbacks: it requires both a light intensity sensor and a temperature one which increase the cost of hardware; the gradient formula is tedious and occupies a lot of processor resources.

The approximate gradient method, based on the P\&O method and the optimal gradient method, uses a first order difference method to replace the gradient calculation of continuous function. It has many advantages such as simple computation, low cost, fast tracking, but also has several defects as "divide by zero error" and wrong reaction to large disturbance.

Through the research on the principle and process of the approximate gradient method, we intend to improve this method, focusing on optimizing the searching strategy. 


\section{Photovoltaic cell characteristics and simulation models}

The output characteristics of PV cell are inseparable with structure and parameters. The equivalent circuit diagram of a single PV cell is shown in Fig. 1.

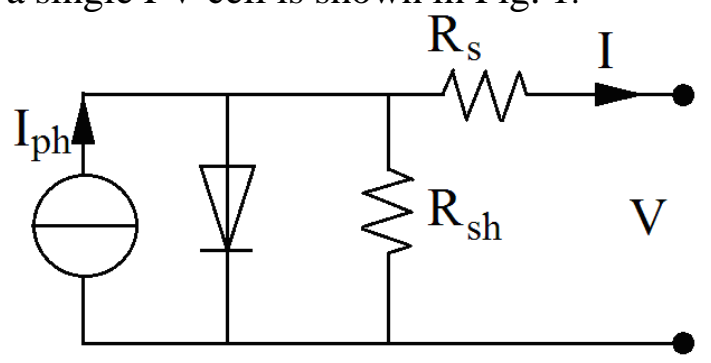

Fig.1. Equivalent circuit diagram of PV cell

The mathematical model of the output current is as follows

$$
I=I_{p h}-I_{r}\left[\exp \left(\frac{V+I R_{s}}{V_{T}}\right)-1\right]-\frac{V+I R_{s}}{R_{s h}}
$$

where $I_{p h}$ is the light generated current, $I_{r}$ is the reverse saturation current of the equivalent diode P-N junction, $V_{T}$ is the thermal voltage and $V_{T}=A K T / q$, A is quality factor of P-N junction, $\mathrm{K}$ is Boltzmann constant, $\mathrm{T}$ is thermodynamic temperature, $\mathrm{q}$ is electron's charge, $R_{s}$ and $R_{s h}$ are respectively series and parallel internal resistances.

Since $R_{s h}$ is a very large number, $\left(V+I R_{s}\right) / R_{s h}$ is so small that it can be neglected. When the environmental conditions are fixed and no reverse breakdown of P-N junction, the output current can be obtained as follows

$$
I=I_{p h}
$$

It is shown in Equation (2) that the P-V curve keeps rising linearly until the reverse breakdown of P-N junction occurs.

Taking Solarex MSX60 as an example, Fig. 2 (a) shows the effect of light intensity on the P-V and current-voltage (I-V) curves at the same temperature as 298K. Fig. 2 (b) shows the effect of temperature on the $\mathrm{P}-\mathrm{V}$ curve at the same light intensity as $1000 \mathrm{~W} / \mathrm{m}^{2}$. It can be observed that current is proportional to light intensity; voltage is inversely proportional to temperature.

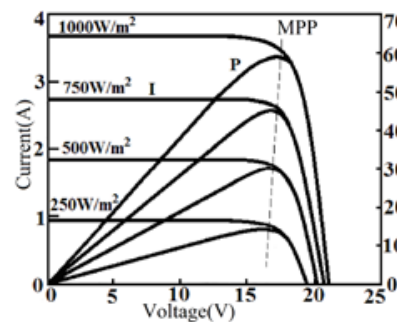

(a)

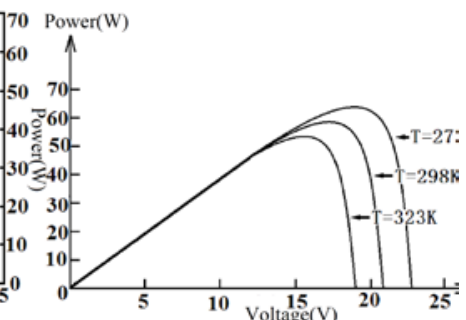

(b)

Fig.2. Solar energy cells output curve. (a) Output characteristics with light intensity. (b) Power with temperature

\section{Principle analysis and method improvement}

Original approximate gradient method measures the output voltage and current, denotes as $V_{k}$ and $I_{k}$, then perturbs the output voltage with a small positive voltage $\left(V^{\prime}\right)$ and takes the new voltage and current as $V_{k+1}$ and $I_{k+1}$. The size of following perturbation step is $\frac{V_{k+1} I_{k+1}-V_{k} I_{k}}{V_{k+1}-V_{k}} V^{\prime}$, and the MPP takes $\frac{V_{k+1} I_{k+1}-V_{k} I_{k}}{V_{k+1}-V_{k}}=0$ as the judgment.

At the beginning of charging, the working point is far from MPP that perturbation steps are too small to approach MPP quickly. To overcome this problem, the CVT method, adjusting the output 
voltage to the reference of MPP $\left(V_{M}\right)$ calculated from the measured $V_{o C}$, is used in the moment before the beginning to narrow the search range. Then the output power $\left(P_{0}\right)$ is calculated according to the output voltage $\left(V_{0}\right)$ and current $\left(I_{0}\right)$ measured.

We select a suitable $V$ 'as the first perturbation step, multiply the output voltage $\left(V_{1}\right)$ and current ( $\left.I_{1}\right)$ to get the power ( $\left.P_{1}\right)$ after the perturbation, and then calculate the difference as $\Delta V=V_{1}-V_{0}$ and $\Delta P=P_{1}-P_{0}$. The formula of perturbation step is simplified to $(\Delta P / \Delta V) V^{\prime}$. Assign $P_{0}=P_{1}$ and $V_{0}=V_{1}$ before the next perturbation for cycle calculations.

Approximate gradient method can automatically change the direction and size of perturbation steps. Comparing the new $P_{1}$ and $V_{1}$ with the assigned $P_{0}$ and $V_{0}$, when $\Delta P>0$, it can be seen that: if $\Delta V<0$, that is, the last perturbation reduced voltage, there would be $(\Delta P / \Delta V) V^{\prime}<0$, thus the next one would also reduce voltage; if $\Delta V>0$, there would be $(\Delta P / \Delta V) V^{\prime}>0$, the next step would increase voltage, too. It means that when $\Delta P>0$, whatever $\Delta V$ is positive or negative, the next step would be in the same direction as the former one. Conversely, when $\Delta P<0$, it would be the opposite.

Obviously, there is $d P / d V=0$ at the MPP in P-V curve. Although $\Delta P / \Delta V$ is very close to $d P$ / $d V$ when $\Delta V$ is minimal, the two points, which make $\Delta P / \Delta V=0$, are lower than true MPP. Moreover, unfortunately, it is not easy to get $\Delta P / \Delta V=0$ in computation of discrete points that there will be oscillation around the MPP for a long time. To reduce the oscillation and power loss, we take a small positive number as $\varepsilon$ to structure a constraint condition as $|\Delta P| \leq \varepsilon$. Then it will stop perturbing when the restriction is met and restart when the balance is disturbed.

When the PV system is subjected to external disturbance, the disturbance is considered as a perturbation of uncontrollable power and voltage. If the disturbance makes $\Delta P>0$, the next step, which would be in the same direction as $\Delta V$, might lead to wrong search direction. Furthermore, when the light intensity changes drastically, power changes greatly while the variation of voltage is very small. The size of next perturbation step will be so large that it may lead to huge tracking error or cross the MPP to generate big swing. What's worse, a big step, crossing the MPP, may make $\Delta P / \Delta V=0$ or $|\Delta P| \leq \varepsilon$ and then stop tracking at the point that is far away from MPP, which means tracking failure. The restriction $(|\Delta P| \leq \varepsilon)$ is not only helpless to the settlement of tracking failure, but also magnify the error rate.

To avoid the failure of tracking, another condition, $|\Delta V| \leq V^{\prime}$, is added to exclude the interference caused by large step perturbation. However, when $|\Delta P| \leq \varepsilon$ and $|\Delta V|>V^{\prime},|\Delta P / \Delta V|$ is so small that even excluding the false MPP, the search speed is still very low even nearly zero. At this time, we change the perturbation step to $V$ 'back into circulation.

If the disturbance only changes power without changing the voltage, that is, $\Delta V=0$, it would lead to "Divide by Zero error". Besides, the original approximate gradient method stops tracking once $\Delta P=0$, and then the next $\Delta V$ will be zero which will also cause "Divide by Zero error". In this case, the PV system will be running in the wrong case until $\Delta V \neq 0$. The judgment $|\Delta P| \leq \varepsilon$ instead of $\Delta P$ / $\Delta V=0$ can avert the second error, but the solution of the first one should be discussed not limited to $\Delta V=0$.

For small disturbance, changing the perturbation step to $V^{\prime}$ when $\Delta V=0$ is desirable. But for the big disturbance, power changes greatly that $V$ 'is not reliable enough to approach the MPP rapidly. By adjusting the voltage to $V_{M}$ and then using the initial step for a new round of tracking, we evade the calculation of the step caused by uncontrollable big disturbance. The critical condition for small and big disturbance is selected as $|\Delta P|=10 \% P_{0}$. Fig. 3 shows the flow chart for improved approximate gradient method. 


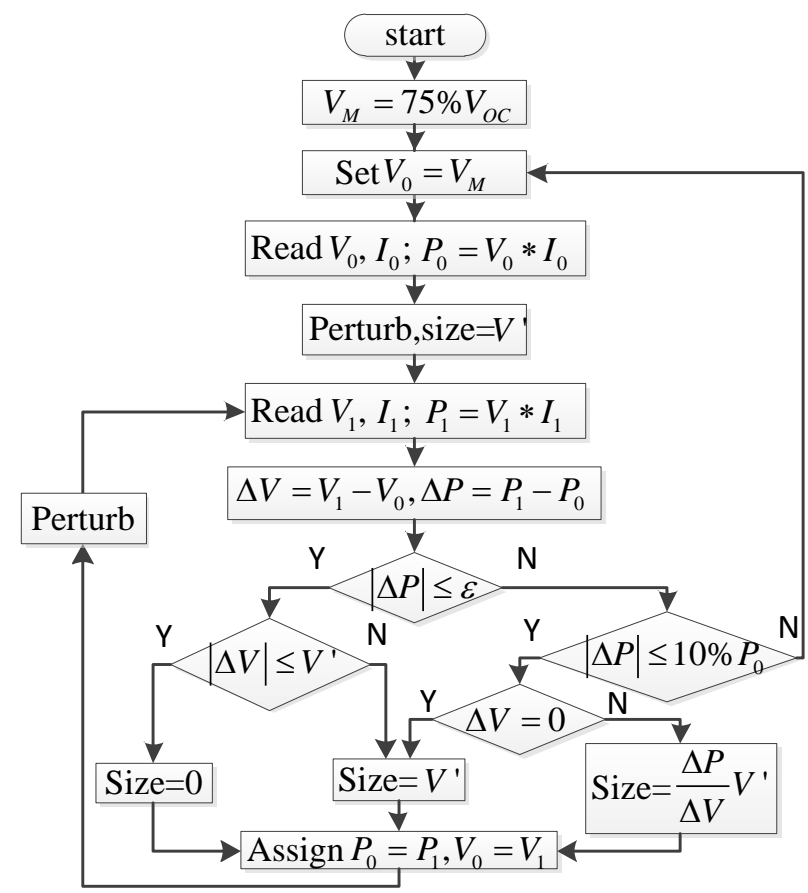

Fig.3. Flow chart for improved approximate gradient method

\section{Simulation and Analysis}

Tab. 1 shows the key specifications of the Solarex MSX60 PV module at a temperature of 298K and a light intensity of $1000 \mathrm{~W} / \mathrm{M}^{2}$, for a case study.

Tab.1 Key specifications of the Solarex MSX60 PV module at 298K, 1000W/M²

\begin{tabular}{|c|c|c|c|}
\hline Open Circuit Voltage & $\mathrm{V}_{\mathrm{oc}}$ & 21.0 & $\mathrm{~V}$ \\
\hline Short Circuit Current & $\mathrm{I}_{\mathrm{SC}}$ & 3.74 & $\mathrm{~A}$ \\
\hline Optimal Voltage & $\mathrm{Vm}$ & 17.1 & $\mathrm{~V}$ \\
\hline Optimal Current & $\mathrm{Im}$ & 3.5 & $\mathrm{~A}$ \\
\hline Maximum Power & $\mathrm{Pm}$ & 59.9 & $\mathrm{~W}$ \\
\hline
\end{tabular}

To verity the performance of the proposed MPPT algorithm, the PV array was simulated with original approximate gradient method and the improved method respectively. In order to reduce the computational time and make the details of power-time (P-t) curves clear, we set the major initial parameters as power of $50 \mathrm{~W}$, voltage of $13.1 \mathrm{~V}$, temperature of $298 \mathrm{~K}$, light intensity of $1000 \mathrm{~W} / \mathrm{M}^{2}$ and data reading time interval of $0.01 \mathrm{~S}$. The constraint conditions were $V^{\prime}=0.15 \mathrm{~V}$ and $\varepsilon=0.1 \mathrm{~W}$. To compare their anti-interference ability, we reduced the temperature to $297 \mathrm{~K}$ and put the light intensity down to $500 \mathrm{~W} / \mathrm{M}^{2}$ at the time of $0.2 \mathrm{~S}$. Then we returned the temperature and light intensity to $298 \mathrm{~K}$ and $1000 \mathrm{~W} / \mathrm{M}^{2}$ at $0.4 \mathrm{~S}$. The P-t curves obtained from the simulation are shown in Fig. 4 where $\mathrm{Pm}$ is the theoretical maximum power.

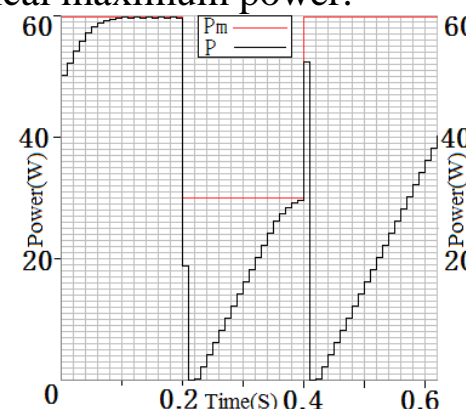

(a)

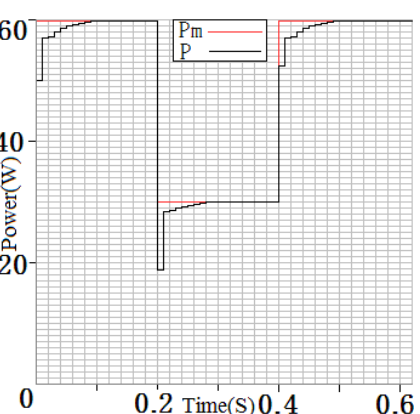

(b)

Fig.4. P-t curves under different approximate gradient methods. (a) Original. (b) Improved. 
Since $V^{\prime}$ is set as a large number, the performance of original method is dismal in Fig. 4 (a) when external conditions change drastically. As shown in Fig. 5, the analysis is as follows

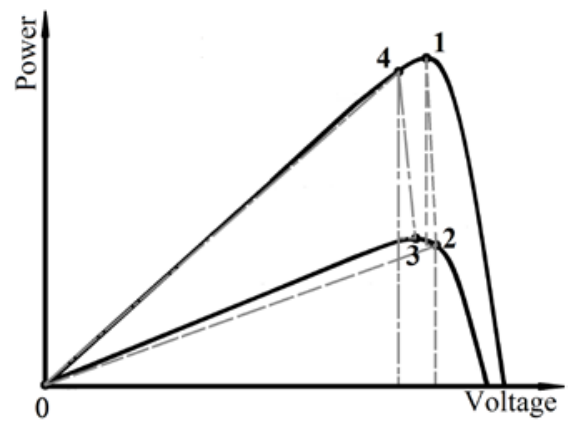

Fig.5. Schematic diagram of power variation

At the moment of $0.2 \mathrm{~S}$, the working point was the point 1 . Light intensity and temperature reduced after the moment, then, from Fig. 2 we know that power reduced a lot but voltage increased a little that the point fell into point 2. Since $|\Delta P / \Delta V|$ was a huge number and $V$ 'was given large, the perturbation reduced voltage with a giant step larger than $V_{O C}$. Fortunately, P-N junction is a unidirectional conductive element that the power point just dropped to 0. Combined with the Equation (2), we found that the power point was in the linear area and kept increasing with a fixed step until reverse breakdown happened. As a similar principle, at $0.4 \mathrm{~S}$, the power point moved from point 3 to point 4 , and then dropped to 0 again.

Comparing the Fig. 4 (a) with Fig. 4 (b), it is clear that the constraint conditions reduced the oscillation around the MPP, meanwhile, when facing a substantial interference, the use of $V_{M}$ to reset search can effectively narrow search range and avoid the tracking errors caused by steps of wrong directions or large sizes.

\section{Conclusion}

Improvements have been proposed for the approximate gradient method by setting constraints to avoid tracking failure of "Divide by zero error", and by using CVT to overcome the inefficiency of small fixed steps away from MPP and to get rid of big steps tracking error. The simulation result proved that the improvements can optimize the search trajectory, reduce search time and power loss, improve the dynamic and static performances of PV generation system.

\section{References}

[1] Barchowsky A, Parvin J P, Reed G F, et al. A comparative study of MPPT methods for distributed photovoltaic generation[C]. IEEE PES Innovative Smart Grid Technologics(ISGT), 2012:1-7.

[2] Parida B, Iniyan S, Goic R. A review of solar photovoltaic technologies[J]. Renewable and Sustainable Energy Reviews, 2011, 15(3):1625-1636.

[3] Stjepanović A, Softić F, Bundalo Z, et al. Solar tracking system and modeling of PV module[C]. Proceedings of the $33^{\text {rd }}$ International Convention MIPRO,2010,105-109.

[4] Koutroulis E, Blaabjerg F. Methodology for the optimal design of transformerless gridconnected PV inverters[J]. Power Electronics IET,2012,5(8):1491-1499.

[5] Bifareti S, Iacovone V, Cina L, et al. Golbal MPPT method for partially shaded photovoltaic modules[C]. IEEE Energy Conversion Congress and Exposition(ECCE),2012:4768-4775. 